\title{
LA REACTIVITE DES ANALYSTES FINANCIERS EN TEMPS DE CRISE AU SEIN DE LA ZONE EURO
}

\author{
Michel Levasseur, Professeur des Universités \\ michel.levasseur@univ-lille2.fr \\ Frédéric Romon, Maître de Conférences \\ frederic.romon@univ-lille2.fr
}

Univ Lille Nord de France, F-59000 Lille, France - SKEMA Business School

Novembre 2010

\begin{abstract}
Résumé : Cet article étudie le lien de causalité entre les variations de cours boursiers et les révisions des prévisions de bénéfice par action formulées par les analystes financiers de la zone Euro lors de la crise financière de 2007-2008. L'étude montre qu'en 2008, les révisions des prévisions avaient un lien moins fort qu'en 2006, année de référence, avec les variations contemporaines de cours et plus fort avec les variations des 3 derniers mois. Ces résultats sont robustes lorsqu'on contrôle les effets du nombre des analystes, du volume des transactions et de l'asymétrie d'information. A partir de ces résultats, nous concluons que les analystes n'ont pas été source d'une information accélérant le krach boursier mais ont ajusté progressivement leur production pour la mettre en accord avec les sentiments du marché.
\end{abstract}

Mots clés : Marché boursier, Prévisions des analystes, Résultat comptable

\begin{abstract}
This article studies the link between stock price changes and analysts' earnings forecasts in the Eurozone during 2007-2008 financial crisis. The study shows that in 2008, change in analysts' earnings forecasts had a smaller link with the contemporenous stock price changes, compared to 2006, which was the benchmark year, but a stronger link with the last three months stock price changes. These results are strong when the effects of the analysts' number, the volume of transactions and the information asymmetry, are controlled. We are concluding, from these results, that the analysts were not responsible for an information which had increased the stock market crash but they had gradually adapted their production to the market needs.
\end{abstract}

Key words : stock market, analyst forecasts, financial analysts, earnings 


\section{$1 \quad$ Introduction}

Les études concernant le comportement des analystes financiers en période de krach boursier sont peu nombreuses. Ang et Ma [2001] ont montré qu'au cours de la crise des marchés asiatiques en 1997, les analystes n'ont pas cédé à la panique et n'ont pas, par des commentaires pessimistes, incité les investisseurs à vendre et précipité ainsi encore plus la baisse. En revanche, ils ont insuffisamment ajusté leurs prévisions et sont restés anormalement optimistes, refusant de prendre en compte à court terme la dégradation forte des économies et la détérioration des situations financières des entreprises. Cet article apporte une contribution supplémentaire à la connaissance du comportement des analystes dans un contexte de crise mondiale (2008), en abordant le cas de pays développés (la zone Euro) et en ayant recours à d'autres méthodes économétriques.

D'un côté, les analystes sont considérés comme des experts, lus par la communauté financière. Leur production est susceptible d'affecter les comportements des investisseurs (Givoly et Lakonishok [1979] ou Imhoff et Lobo [1984], pour des contributions pionnières). Sont-ils toujours en période de krach boursier une source d'informations réellement nouvelles pour les marchés ? Dans le cadre d'une étude des consensus, nous nous attachons à une première question. Durant les mois de crise, les cours boursiers ont-ils reflété sans délai les informations apportées par les consensus construits à partir des prévisions de bénéfice des analystes? D'un autre côté, lors d'un krach, les fortes variations des cours traduisent des changements importants dans les anticipations des opérateurs. Quelle est alors la réactivité des analystes pour faire le tri au sein de ce flot d'informations et élaborer leurs propres prévisions de bénéfice? Nous abordons alors une seconde question. Ces consensus ont-ils incorporé rapidement les informations prises en compte par les marchés boursiers?

Notre échantillon est composé de 727 sociétés cotées appartenant à la zone euro. Pour toutes ces sociétés, nous disposons du consensus des prévisions de bénéfice par action fournies par la base I/B/E/S de Thomson Financials sur la période 2006-début 2009. Nous réalisons une série de tests de causalité de Granger pour analyser les relations entre les variations de cours boursier et les révisions de prévision de bénéfice par action dans un sens et dans l'autre. Les estimations économétriques sont effectuées dans le cadre de panels avec effets fixes. Nous complétons cette étude par une série de résultats complémentaires et des tests de robustesse. Nos tests de causalité de Granger apportent des réponses claires aux questions que nous avons posées. En premier lieu, sur la période de crise étudiée, nous rejetons l'hypothèse selon laquelle les révisions de prévision de bénéfice par action du consensus incorporent sans délai les informations prises en compte par les marchés boursiers. Nombre d'analystes n'actualisent leurs prévisions qu'avec retard et en suivant les mouvements du marché. En second lieu, nous ne mettons en évidence aucune relation positive et significative entre les variations de cours et les révisions passées des prévisions de bénéfice par action.

Cet article propose des tests empiriques, à notre connaissance, nouveaux sur les relations entre les productions des analystes financiers et les fluctuations des cours boursiers en période de krach boursier. Nos résultats sont cohérents avec les contributions qui affirment que les rapports des analystes servent avant tout à synthétiser et à mettre en perspective une information complexe à l'usage d'un public large (Kothari et al. [2009]). En période de crise, l'une de leurs tâches est ainsi de traduire sous forme de prévisions de résultats le flot des 
informations contenues dans les cours boursiers. Nous devons insister cependant sur trois limites liées à la méthodologie choisie :

(1) les relations testées sont de l'ordre de la corrélation et ne permettent pas d'expliciter des mécanismes de causalité

(2) notre approche ne permet pas d'identifier les analystes qui réagissent avec le plus de retard et d'expliciter les raisons de leurs comportements

(3) nos résultats ne sont pas contradictoires avec le fait que certains analystes puissent fournir une information privée utile aux gérants de portefeuille.

L'article se poursuit ainsi. La section 2 propose une revue de la littérature et un exposé des hypothèses. La section 3 présente les données et décrit leurs principales caractéristiques. La section 4 rappelle la méthode utilisée. La section 5 discute les résultats empiriques obtenus. La section 6 expose des tests de robustesse et des extensions. Enfin, la section 7 conclut.

\section{Revue de la littérature et hypothèses}

Les révisions des prévisions de bénéfice par action sont les productions les plus suivies des analystes. Jouent-elles un rôle dans la diffusion de l'information au sein des marchés boursiers ? Cette interrogation est au cœur de nombreux tests depuis les années 70 (Griffin [1976], Givoly et Lakonishok [1979], [1980], Imhoff et Lobo [1984], par exemple). Elle prend une importance particulière en période de crise financière où les opérateurs paraissent avoir beaucoup de difficultés à établir un prix satisfaisant pour les titres. Les analystes apportent-ils en ces périodes troublées des repères utiles ou ne font-ils que s'adapter aux évolutions des marchés?

\subsection{Les analystes transmettent une information pertinente au marché}

Un courant de la littérature soutient que les analystes sont source de nouvelles qui ne sont pas encore incorporées dans les prix de marché. Les révisions de prévision de bénéfice ont alors un intérêt pour les investisseurs puisqu'ils révèlent alors un changement d'opinion des analystes. Green [2006] et Chang et Chan [2008], par exemple, mettent en évidence que ces études peuvent être utiles à la gestion active des portefeuilles. Ainsi, pour Gleason et Lee [2003], le marché s'ajuste lentement à l'information venant des révisions de prévision de bénéfice par les analystes, les annonces successives se comportant comme des catalyseurs dans la formation des cours. Dans le contexte d'une crise financière, le rôle des analystes en matière d'information des investisseurs s'en trouverait renforcé. Les professionnels accorderaient alors moins de poids aux résultats passés car leur contenu informatif est faible (cet argument a été développé pour les sociétés réalisant des pertes par Sedor [2002]). Confrontés au risque de commettre d'importantes erreurs de prévision, ils intensifieraient leur recherche. Plus généralement, Barron et al. [2008] montrent que suite à des erreurs de prévision importantes, les analystes augmentent non seulement le nombre de leurs estimations mais les fondent aussi sur plus d'information privée. En période de crise, trois types d'arguments viennent soutenir le rôle des analystes : (i) les pertes étant moins persistantes (Freeman and Tse [1992], Basu [1997]), le lien entre résultat passé et valeur est plus ténu, (ii) 
les firmes répugnant à communiquer une information négative (Waymire [1985], Miller [2002]), l'information publique se raréfie, (iii) la motivation des analystes est plus grande car ils craignent la dégradation de leur réputation (Stickel [1989], Mikhail et al. [1999]). De leur côté, Frankel et al. [2006] soutiennent que le contenu informatif des révisions des analystes est plus élevé si la nouvelle est négative que si elle est positive car dans ce dernier cas, elle a déjà été largement prise en compte par le marché.

En résumé, en période de crise financière, les révisions des prévisions des bénéfices par action devraient donner des indications utiles au marché. Si elles sont rapidement et largement communiquées, elles devraient être reflétées immédiatement dans les cours boursiers.

\subsection{Les productions des analystes s'ajustent aux mouvements du marché}

A l'opposé, un autre courant de la littérature soutient que les analystes sont source de peu de nouvelles qui ne soient pas déjà incorporées dans les prix de marché (Kothari et al. [2009]). Ces auteurs ne nient pas l'importance de leur travail dans la recherche d'information mais soulignent la force des contraintes auxquelles ils sont soumis au sein des maisons de courtage et des banques d'affaires (Lin and McNichols [1998], Michaely and Womack [1999], Barber et al. [2007], Kothari et Kolasinski [2008], Brown [2009]). Ces conflits d'intérêt affectent les prévisions (Chen et Jiang [2006]), même si la présence d'investisseurs institutionnels est de nature à atténuer leurs effets (Ljungqvist et al. [2007]). Kothari et al. [2009] décrivent les analystes comme avant tout des producteurs d'études rétrospectives qui présentent l'intérêt de synthétiser et de mettre en perspective une information complexe à l'usage d'un public large.

En résumé, si les analystes effectuent avec célérité leurs révisions en fonction des mouvements du marché, leur production reflète la totalité des informations contenues dans les prix, y compris en période de crise financière. Mais, les révisions à effectuer y sont aussi plus nombreuses et d'une plus grande amplitude, vu les modifications dramatiques des environnements. Les contraintes qui pèsent sur les analystes peuvent les rendre plus difficiles à proposer. Alors ils pourraient être plus lents à apparaître.

\subsection{Hypothèses}

Dans cette étude, nous nous intéressons aux prévisions de bénéfice par action parce que leurs révisions sont les plus suivies (Givoly et al. [2009). Comme les comportements des analystes à la source de ces consensus sont divers, nous retenons deux hypothèses non mutuellement exclusives. D'une part, nous supposons qu'en période de crise financière, si certains analystes transmettent une information pertinente, les forces du marché sont suffisamment fortes pour qu'elle soit immédiatement prise en compte. En conséquence, nous avançons que les variations de cours boursier ne sont pas corrélées aux révisions passées des prévisions de bénéfice par action. D'autre part, en reprenant la seconde argumentation selon laquelle les productions des analystes s'ajustent aux mouvements du marché, nous proposons l'hypothèse suivant laquelle en période de krach boursier, la pression est suffisamment forte pour pousser les analystes à réagir immédiatement et à actualiser sans délai leurs indications. Si tel est le cas, les révisions de prévision de bénéfice par action ne sont pas corrélées aux variations passées de cours boursier. 


\section{$3 \quad$ Les données}

En dehors des USA, les pays de la zone euro, ont l'avantage d'offrir un échantillon de taille importante, homogène au point de vue monétaire (pas de problèmes liés au change) et composé d'entreprises opérant dans un cadre comptable harmonisé ${ }^{1}$. Ce dernier point est important car les différents référentiels comptables peuvent affecter le suivi des entreprises par les analystes (Bae et al. [2008]). L'échantillon est aussi homogène du point de vue de la participation des investisseurs individuels qui est certainement moins élevée qu'aux USA. La très grande majorité des titres (62\%) est traitée sur l'une des deux grandes bourses de l'Europe continentale : NYSE Euronext et Deutsche Börse Group. Enfin, la base de données I/B/E/S de Thomson Financials offre une bonne couverture pour ces titres.

Une crise financière de grande ampleur est à la fois un évènement difficile à anticiper par les analystes et de nature à remettre en cause l'ensemble des prévisions réalisées. Le krach boursier de 2007/2008 est mondial et particulièrement violent. L'indice S\&P 500 a baissé sur 6 mois de $42,7 \%$ entre août 2008 et février 2009 alors qu'il n'avait baissé que de $20.6 \%$ entre mai et novembre 1987, de $19.2 \%$ entre septembre 2000 et mars 2001 et de $28.9 \%$ entre mars et septembre 2002. La période de crise ${ }^{2}$ étudiée est comprise entre avril 2008 et janvier 2009. La période d'avril 2006 à janvier 2007 a été retenue comme période de contrôle. Pour faciliter les comparaisons entre les prévisions de résultats, nous nous sommes limités aux entreprises clôturant leur compte au 31 décembre (plus de $85 \%$ de notre population). Toutes les prévisions sont alors synchrones si on les date par rapport à la clôture de l'exercice. Comme la plupart annoncent leurs résultats sur la période février-avril (plus de $97 \%$ de notre population), nous avons éliminé ces mois de nos périodes d'étude. En effet, l'annonce de résultats passés est un événement particulier dans la gestion des prévisions de bénéfice par action. En conséquence, les tests économétriques sont conduits sur deux périodes de 9 mois consécutifs, avril 2006 - janvier 2007, référée par la suite comme année 2006 et avril 2008 janvier 2009, référée comme année 2008.

\subsection{Les variables}

Dans cette recherche, nous étudions le comportement des analystes à travers les consensus publiés par $\mathrm{I} / \mathrm{B} / \mathrm{E} / \mathrm{S} /$. L'intérêt d'un consensus est de contenir les réactions de tous les analystes présents dans cette base, qu'ils appartiennent à de grandes ou à de petites institutions, qu'ils soient fortement spécialisés ou non, qu'ils soient considérés comme leaders ou non. L'une des limites importantes de cette source est de n'être alimentée que par des analystes essentiellement «sell-side». Néanmoins, ces données sont largement publiées et sont susceptibles d'affecter les comportements d'un grand nombre d'acteurs.

Parmi toutes les prévisions disponibles de bénéfice par action, nous retenons les prévisions pour l'exercice suivant et non l'exercice en cours car elles sont nombreuses, fréquemment révisées et souffrent moins des effets de changement de biais (optimisme au départ,

\footnotetext{
${ }^{1}$ Depuis le 1/01/2005, toutes les sociétés européennes cotées en bourse doivent établir leurs comptes consolidés en normes IFRS.

${ }^{2}$ Les extractions de données ont été réalisées pour leur plus grande part en novembre 2009 et pour les réalisations de bénéfice par action de l'exercice 2009 au cours du mois de juin 2010.
} 
pessimisme à la fin) que les prévisions pour l'exercice en cours (Bartov et al. [2002]). Par ailleurs, Schipper [1991], Clement et Tse [2003] soutiennent que les investisseurs réagissent plus fortement aux prévisions émises plus tôt et privilégient la récence à la précision. Ainsi, en 2006, nous retenons les prévisions pour l'exercice 2007 et en 2008 celles pour l'exercice 2009. Il s'agit du consensus moyen publié par $\mathrm{I} / \mathrm{B} / \mathrm{E} / \mathrm{S}^{3}$ le $1^{\text {er }}$ jour de chaque mois. Pour faciliter les comparaisons, les bénéfices réalisés sont ceux publiés par la même base $\mathrm{I} / \mathrm{B} / \mathrm{E} / \mathrm{S}$ de Thomson (IBH.EPSActualValue).

La variable étudiée est la variation d'un mois sur l'autre de ce consensus moyen qui représente l'information nouvelle retraitée par les analystes. Aussi, nous considérons que la variation $\mathrm{du}$ consensus moyen entre deux mois représente principalement l'apport informationnel du mois de départ ${ }^{4}$. Comme les bénéfices par action diffèrent d'une société à l'autre par des facteurs de taille importants, nous avons corrigé les variations en les divisant par le cours boursier de l'action début 2006. Enfin, la date de publication des résultats provient de l'item IBH.EPSActualReportDate, disponible dans la base I/B/E/S. Lorsque l'information est manquante, une date approchée est retenue en reprenant le mois de publication des autres années. Les variations ajustées de cours boursiers ${ }^{5}$ ont été calculées à partir des cours de fin de mois publiés dans la base Thomson Financials ${ }^{6}$.

\footnotetext{
${ }^{3}$ II s'agit dans la base I/B/E/S de Thomson Financials soit des items IBH.EPSMeanFYR2, soit des items IBH.EPSMeanFYR3. II se peut que la prévision concernant l'année à venir corresponde à la deuxième (FYR2) ou à la troisième (FYR3) prévision disponible. Par exemple, début 2006, la première prévision (FYR1) concerne 2005 et la troisième (FYR3) 2007. Fin 2006, la première prévision (FYR1) concerne 2006 et la seconde (FYR2) 2007. Les dates de bascule d'un exercice à l'autre varient selon les entreprises. Nous les avons contrôlées et construit une base homogène. Les mesures de dispersion des prévisions correspondent de même aux items IBH.EPSStdDeviationFYR2 ou IBH.EPSStdDeviationFYR3.

${ }^{4}$ Par exemple, la variation entre les prévisions au $1{ }^{\text {er }}$ mai et au $1{ }^{\text {er }}$ juin 2006 est supposée correspondre à l'information apportée par les analystes durant le mois de mai 2006.

${ }^{5}$ Des opérations de capital et des dividendes détachés.

${ }^{6}$ Ils correspondent à l'item TF. PriceClose sur la base Thomson Financials et pour la fréquence mensuelle, sont des cours de fin de mois.
} 


\subsection{L’échantillon}

Nous avons recensé l'ensemble des entreprises actives et non actives présentes sur la base Thomson Financials en novembre 2009 pour tous les pays appartenant à cette date à la zone Euro. Le nombre total de firmes s'élève à 5748 avec deux pays dominants : la France (1 584) et l'Allemagne (1 468). Nous avons éliminé l'ensemble des entreprises bancaires, financières et immobilières ${ }^{7}$ ( 1343 ), les entreprises pour lesquelles I/B/E/S ne fournit pas d'information sur le bénéfice par action réalisé (2 233). Nous avons limité l'étude aux seules entreprises dont l'exercice comptable s'achève au 31 décembre pour disposer de prévisions d'âge homogène : 320 entreprises ont été de ce fait perdues. Il manquait pour 341 entreprises des informations financières ou comptables. Enfin, le calcul des variations de prévision de bénéfice par action ont réduit encore la taille de l'échantillon. Pour disposer d'un suivi continu sur les 3 années considérées (2006-2008), il faut se restreindre à l'étude de 727 entreprises.

$$
\text { < insérer le Tableau 1> }
$$

La répartition finale par pays est proche de l'initiale. En reprenant la classification de Fama et French en 49 secteurs, nous avons vérifié que l'échantillon recouvre des spectres larges d'activité ${ }^{8}$. Il n'existe pas de concentration particulière des entreprises d'un pays dans un secteur particulier.

\subsection{Les statistiques descriptives}

Le Tableau 2 présente les statistiques descriptives portant sur les principales variables de l'étude. Les capitalisations boursières ont fortement chuté entre 2006 et 2008 (- 35\% par rapport à la moyenne et - 50\% par rapport à la médiane). L'échantillon est composé d'entreprises de taille différente (la capitalisation par décile varie progressivement de 23 à 12740 millions d'euros en 2008). Les entreprises sont plus petites en Belgique et en Grèce que dans les autres pays mais il n'y a pas de lien direct entre la taille des pays et la capitalisation moyenne des entreprises.

$$
<\text { insérer le Tableau } 2>
$$

Le nombre moyen de prévisions de bénéfice par action (calculé sur une base mensuelle) est stable sur les deux années: il passe de 9,6 en 2006 à 10,3 en 2008 (différence non significative). Le nombre moyen de prévisions augmente avec la capitalisation boursière des sociétés. En 2006 (2008), la moyenne pour le premier et le dernier décile est respectivement de $2,2(2,3)$ et $24,4(25,4)$. Le suivi par les analystes est bien fonction de la taille de l'entreprise.

\footnotetext{
${ }^{7}$ Secteurs 45 à 49 dans la classification de Fama et French.

${ }^{8}$ Les tableaux sont disponibles auprès des auteurs et ne sont pas repris ici pour des raisons de concision.
} 
Des taux de révision des prévisions de bénéfice ont été calculés pour chaque entreprise et chaque mois en divisant le nombre des révisions effectués par les nombres de prévisions. Nous observons une augmentation du taux moyen de révision de $27 \%$ en 2006 à $33 \%$ en 2008 (différence significative au seuil de 1\%). Cet accroissement est constaté dans tous les déciles de taille d'entreprises et dans tous les pays. Ainsi, l'arrivée de la crise n'a pas provoqué une augmentation du nombre de suivi des entreprises (plus de prévisions) mais a occasionné en moyenne une augmentation du nombre de révisions.

Le Tableau 3 (panel A) présente des estimations sur la précision (moyenne des erreurs absolues) et le biais (moyenne des erreurs) des prévisions de bénéfice par action selon l'âge de la prévision (20 mois ou 14 mois) et le nombre d'analystes suivant l'entreprise (moins de 5 , entre 5 et 10 , plus de 10 analystes). A 20 mois ou à 14 mois, la précision est meilleure en 2006 qu'en 2008. Ainsi, à 20 mois, elle est de 3,31\% en 2006, contre 7,07\% en 2008. Ces différences sont toutes statistiquement significatives au seuil de $1 \%$. Cependant, bien que la prévision soit de meilleure qualité en 2006, elle ne s'améliore pas en se rapprochant de la date d'annonce du résultat. La précision moyenne de la prévision à 20 mois est de 3,31\% en 2006. Six mois plus tard (14), elle est de $2,86 \%$ (la différence n'est pas statistiquement significative au seuil de $5 \%$ ). En revanche, pour 2008, plus la date d'annonce du résultat approche, plus la précision s'améliore. Elle passe de 7,07 \% (20 mois) à 4,77 \% (14 mois) (différence statistiquement significative au seuil de $1 \%$ ). Nous constatons une amélioration de la précision de la prévision avec le nombre d'analystes suivant les titres. En 2006, à 20 mois, elle passe de 4,56\% pour les entreprises les moins suivies à 2,24\% pour les entreprises les plus suivies. En revanche, si le biais moyen de la prévision décroît avec la proximité de la date d'annonce, il n'y a pas de relation monotone observée avec le nombre d'analystes suivant le titre.

$<$ insérer le Tableau $3>$

Le Tableau 3 (Panel B) présente pour les mois de mai, septembre et janvier et selon le nombre d'analystes, les révisions des prévisions de bénéfice par action. Le nombre moyen de révisions augmente mécaniquement avec le nombre d'analystes (les taux de révision étant souvent peu différents). On n'observe pas d'augmentation systématique du taux des révisions entre 2006 et 2008. Le taux de révision moyen des prévisions est voisin en mai 2006 et mai $2008(23,88 \%$ et $26,45 \% \%)$ mais cette différence n'est pas significative au seuil de $5 \%$. Il est plus faible en septembre 2008 (25,53\%) qu'en septembre 2006 (30,44\%), mais plus élevé en janvier 2009 (47,55\%) qu'en janvier 2007 (32,82\%), tous ces écarts étant significatifs au seuil de $1 \%$. Tout au plus, peut-on supposer que les analystes aient attendu un peu plus longtemps en période de crise pour réviser leur prévision. 


\section{$4 \quad$ Méthode}

Nous cherchons à vérifier l'absence de relation de causalité (au sens de Granger) entre les révisions mensuelles de prévisions effectuées par les analystes financiers et les variations passées des cours des actions concernées. Nous nous inspirons de la méthodologie développée par Hurlin [2005] pour les données de panel à effet fixe.

Considérons le modèle suivant :

$y_{i, t}=\sum_{k=1}^{p} \gamma_{k} \cdot y_{i, t-k}+\sum_{k=0}^{p} \beta_{k} \cdot x_{i, t-k}+\alpha_{i}+\delta_{t}+\varepsilon_{i, t} \quad$ Eq. 1

L'indice i dénote les firmes présentes dans les coupes instantanées, $\mathrm{t}$ les mois considérés et $\mathrm{p}$ les décalages pris en compte. $\varepsilon_{i, t}$ sont des bruits blancs individuels. $y_{i, t}$ et $x_{i, t}$ satisfont les conditions de stationnarité en covariance ${ }^{9}$. Les coefficients $\gamma_{k}$ et $\beta_{k}$ sont supposés constants dans le temps et identiques pour toutes les firmes. Les coefficients $\alpha_{i}$ et $\delta_{t}$ mesurent les effets fixes par firme et de contraste. La valeur contemporaine de $x_{i, t}$ a été introduite pour prendre en compte la corrélation contemporaine avec la variable $y_{i, t}$.

L'hypothèse de non causalité (homogène) se réfère au cas où il n'y a pas de causalité linéaire entre la variable dépendante $y_{i, t}$ et la variable explicative $x_{i, t}$ dans la population étudiée. Les hypothèses nulle et alternative sont dans ce cas :

$$
\begin{gathered}
H_{0}: \beta_{k}=0, \forall k \in[1, p] \\
H_{a}: \exists k, \quad \beta_{k} \neq 0
\end{gathered}
$$

Sous ces hypothèses, nous procédons en trois étapes pour mener ce test, comme il est suggéré par Hurlin [2005] :

\section{Etape 1 :}

Nous estimons par les moindres carrés ordinaires le modèle complet suivant (les $\mathrm{T}$ sont calculés à partir d'écart-types estimés avec des clusters par firme et par période, voir Petersen [2009] et Thompson [2009]) :

$y_{i, t}=\sum_{k=1}^{3} \gamma_{k} \cdot y_{i, t-k}+\sum_{k=0}^{3} \beta_{k} \cdot x_{i, t-k}+\alpha_{i}+\delta_{t}+\varepsilon_{i, t} \quad$ Eq. 2

Nous avons limité le nombre de décalages à 3 mois puis nous calculons la somme des résidus au carré $\left(R S S_{1}\right)$.

\footnotetext{
${ }^{9}$ Un processus $z_{t}$ stochastique est faiblement stationnaire ou stationnaire en covariance s'il satisfait les 3 conditions : $E\left[z_{t}\right]$ est indépendante de $t, \operatorname{Var}\left[z_{t}\right]$ est finie et indépendante de $t, \operatorname{Cov}\left[z_{t}, z_{s}\right]$ est une fonction finie de t-s (voir Greene [2000], p.752).
} 


\section{Etape 2 :}

Nous estimons ensuite le modèle réduit suivant :

$y_{i, t}=\sum_{k=1}^{3} \gamma_{k} \cdot y_{i, t-k}+\beta_{0} \cdot x_{i, t}+\alpha_{i}+\delta_{t}+\varepsilon_{i, t} \quad$ Eq. 3

Puis, nous calculons la somme des résidus au carré $\left(R S S_{2}\right)$.

\section{Etape 3 :}

Enfin, nous mesurons la statistique suivante :

$$
S=\frac{\left(R S S_{0}-R S S_{1}\right) / p}{R S S_{1} /(N-2 p-1)} \quad \text { Eq. } 4
$$

où $\mathrm{p}$ est la longueur du décalage (3 dans la spécification utilisée) et $\mathrm{N}$ le nombre d'observations. S suit asymptotiquement une distribution $F(p, T-2 p-1)$.

Les estimations ont été effectuées année par année sur une période de 9 mois. Les variables considérées sont la révision de la prévision normée du bénéfice par action par les analystes $\left(V E_{i, m}\right)$ et les variations mensuelles des cours $\left(R_{i, m}\right)$.

$$
\begin{aligned}
& \boldsymbol{R}_{i, t}=\sum_{k=1}^{p} \gamma_{k} \cdot \boldsymbol{R}_{i, t-k}+\sum_{k=0}^{p} \boldsymbol{\beta}_{k} \cdot \boldsymbol{V} \boldsymbol{E}_{i, t-k}+\boldsymbol{\alpha}_{i}+\boldsymbol{\delta}_{t}+\boldsymbol{\varepsilon}_{i, t} \\
& \boldsymbol{V} \boldsymbol{E}_{i, t}=\sum_{k=1}^{p} \gamma_{k} \cdot \boldsymbol{V} \boldsymbol{E}_{i, t-k}+\sum_{k=0}^{p} \boldsymbol{\beta}_{k} \cdot \boldsymbol{R}_{i, t-k}+\boldsymbol{\alpha}_{i}+\boldsymbol{\delta}_{t}+\varepsilon_{i, t}
\end{aligned}
$$

Les activités de révision par les analystes ne sont pas régulières mais sont influencées par le calendrier comptable. Stickel [1989] relève que les annonces de résultat sont suivies par une activité soutenue de révision des prévisions par les analystes financiers. Barron et al. [2002] font valoir que l'explication réside dans le fait que ces derniers agissent en qualité de “processeurs d'information. L'étude tient compte de cette saisonnalité. Onze ${ }^{10}$ variables muettes $\delta_{t}$ sont introduites pour tenir compte de la date de révision. Elles prennent la valeur 1 si l'observation est réalisée $t$ mois avant la prochaine annonce de résultat, zéro sinon. L'indice t vaut 1 , par exemple, si l'observation est faite le mois qui précède l'annonce et 11 lorsqu'on est 11 mois avant la prochaine publication (et de fait un mois après la dernière, du moins si le calendrier est régulier). Enfin, l'inclusion d'un effet fixe $\alpha_{i}$ par firme permet de contrôler les effets constants des variables omises à travers le temps et élimine une des sources principales de corrélation des observations successives pour chaque firme.

\footnotetext{
${ }^{10}$ Autant de variables muettes que de mois dans l'année moins un.
} 


\section{$5 \quad$ Résultats}

Cette section décrit les résultats obtenus sur notre échantillon global. Dans un premier paragraphe, nous étudions la période de crise et la comparons à celle de contrôle. Dans un deuxième, nous estimons directement les différences entre les deux périodes.

\subsection{Année de crise 2008 versus année de contrôle 2006}

Le Tableau 4 rapporte les résultats des estimations des modèles complets (Eq.2) et réduits (Eq.3) lorsque la variable dépendante est la variation du cours boursier sur le mois (Panel A) ou la révision mensuelle de la prévision de bénéfice par action (Panel B). Par parcimonie, les coefficients associés aux effets fixes ou de contraste n'ont pas été repris. Les statistiques T ont été calculées à partir d'écart-types estimés avec des clusters par firme et par période (voir Petersen [2009] et Thompson [2009]).

$$
\text { < insérer le Tableau } 4>
$$

Les résultats du panel A ne mettent en évidence aucune relation significative entre les variations des cours sur un mois et les révisions de prévision de bénéfice par action au cours des mois qui précèdent. Les signes des coefficients sont de plus négatifs en 2006 et 2008 . Ce résultat n'est pas surprenant puisqu'il suggère que les informations prises en compte par les analystes sont soit déjà incorporées dans les cours ou le sont au cours du même mois, ce qui est conforme à l'hypothèse d'efficience des marchés. On peut remarquer que le coefficient associé à la révision contemporaine dans le modèle réduit est positif et significatif en 2006 mais ne l'est plus en 2008. Cette observation n'est pas cohérente avec l'hypothèse suivant laquelle en période de crise, les analystes fournissent au marché une information nouvelle qui est prise rapidement en compte.

Les tests de causalité de Granger décrits au panel B sont significatifs pour les deux années 2006 et 2008. Ils suggèrent que les informations reflétées dans les variations de cours des 3 derniers mois ont un contenu significatif pour expliquer les révisions de bénéfice par les analystes. Par ailleurs, comme au panel A, on peut observer que l'association contemporaine entre les deux variables disparaît en 2008. Le consensus moyen des prévisions de bénéfice réagit avec retard aux informations incorporées dans les cours boursiers, particulièrement en période de crise.

\subsection{Estimation des différences dans les effets des variables indépendantes entre 2006 et 2008}

Le 
Tableau 5 fournit une étude des différences constatées entre une année sans crise (2006) et une année de crise financière (2008), au niveau des coefficients associés aux valeurs décalées des variables explicatives du modèle décrit par l'équation (Eq.7). Pour mettre en évidence ces différences, nous estimons dans un premier temps par les moindres carrés ordinaires le modèle (Eq. 7) sur l'échantillon comprenant à la fois les données de 2008 et de 2006 avec $\theta$ égal à 1 en 2008 et 0 sinon puis calculons la somme des résidus au carré $\left(R S S_{4}\right)$.

$\boldsymbol{y}_{i, t}=\sum_{k=1}^{3} \gamma_{k, 1} \cdot y_{i, t-k}+\sum_{k=1}^{3} \gamma_{k, 2} \cdot \theta \cdot y_{i, t-k}+\sum_{k=0}^{3} \beta_{k, 1} \cdot x_{i, t-k}+\sum_{k=0}^{3} \beta_{k, 2} \cdot \theta \cdot x_{i, t-k}+$ $\alpha_{i}+\delta_{t}+\varepsilon_{i, t}$

\section{(Eq. 7)}

Dans un deuxième temps, nous estimons le modèle (Eq. 8) toujours sur l'échantillon global et calculons la somme des résidus au carré $\left(R S S_{5}\right)$.

$y_{i, t}=\sum_{k=1}^{3} \gamma_{k, 1} \cdot y_{i, t-k}+\sum_{k=1}^{3} \gamma_{k, 2} \cdot \theta \cdot y_{i, t-k}+\sum_{k=0}^{3} \beta_{k, 1} \cdot x_{i, t-k}+\beta_{0,2} \cdot \theta \cdot x_{i, t}+\alpha_{i}+$ $\delta_{t}+\varepsilon_{i, t}$

(Eq. 8)

Pour tester le caractère globalement significatif des coefficients $\beta_{k, 2}$, nous mesurons la statistique $\frac{\left(R S S_{5}-R S S_{4}\right) / 3}{R S S_{4} /(N-14)}$ qui suit asymptotiquement une distribution $\mathrm{F}(3, \mathrm{~N}-14)$ où $\mathrm{N}$ désigne le nombre d'observations.

Les tests reportés au Panel A du 
Tableau 5 concernent les relations entre les révisions mensuelles des prévisions de bénéfice par le consensus des analystes financiers et les variations mensuelles des cours boursiers. La révision de la prévision de bénéfice en 2006 est significativement associée à la variation contemporaine du cours boursier mais ne l'est plus en 2008 (la somme des coefficients associés à $\mathrm{R}_{\mathrm{i}, \mathrm{m}}$ et à $\mathrm{DR}_{\mathrm{i}, \mathrm{m}}: 0.017-0.020$ n'est pas significativement différente de zéro). Les variations de cours décalées d'un, deux et trois mois $\left(R_{i, m-1}, R_{i, m-2}\right.$ et $\left.R_{i, m-3}\right)$ sont significativement et positivement associées aux révisions. De plus, les variables composites (produits de ces variations et d'une variable muette représentant l'année de crise) sont également toutes positives et globalement significatives. La relation entre les variations passées des cours boursiers et les révisions de consensus est fortement renforcée en période de crise puisque la valeur de tous les coefficients s'en trouve plus que doublée. En revanche, le test conduit sur la relation de sens opposé entre ces deux variables n'aboutit à aucun résultat significatif. Ceci suggère qu'en période de crise, le contenu informationnel que reflètent les variations de cours est pris en compte avec retard par les analystes au fur et à mesure des révisions de leurs prévisions, le phénomène étant accentué en période de crise.

<insérer le 
Tableau 5>

\section{Extensions et tests de robustesse}

Nous proposons dans ce qui suit deux études complémentaires : d'abord un contrôle des effets potentiels de variables considérées dans la littérature comme explicatives du comportement des analystes financiers, enfin un traitement des probabilités d'absence de révisions des prévisions.

\subsection{Impact des facteurs}

Notre échantillon est relativement hétérogène. Il est composé d'entreprises de nationalité différente, de taille variée ou encore appartenant à des secteurs divers. C'est pourquoi nous vérifions que nos résultats ne proviennent pas de sous-populations particulières. Pour ce faire, nous segmentons notre échantillon en fonction de 6 variables susceptibles d'être associées à des comportements différents des analystes en matière de suivi de ces firmes. Comme les prévisions de bénéfice par action sont des moyennes de données fournies par des groupes d'analystes de composition variée, nous pensons que l'intensité du suivi, mesurée par le nombre d'analystes (1) fournissant une prévision peut affecter la qualité des mesures utilisées (Lang et Lundholm [1996], Barth et al. [2001], par exemple). La production des analystes peut varier en qualité et en quantité avec les volumes de transaction (2) des actions, parce que, par exemple, les courtages générés en sont fonction, ou encore qu'il est plus facile de tirer un profit d'une information supérieure sur un marché où les ordres sont nombreux (Frankel et al. [2006]). Si les analystes améliorent l'environnement informationnel, leurs efforts sont plus intenses pour les firmes qui sont difficiles à suivre. Si les performances des analystes sont évaluées en fonction de la précision de leurs prévisions, alors ils peuvent être découragés de suivre les titres les plus risqués. La dispersion de leurs prévisions est fréquemment associée à l'asymétrie d'information entourant les résultats attendus (Brown et al. [1987], Wiedman [1996], Bryan et Tiras [2007]). Pour rendre compte de cette asymétrie d'information nous avons préféré la mesure (3) de BKLS (Barron et al. [1998]) qui tient compte à la fois du nombre des analystes, de la dispersion et de la précision des prévisions. Par ailleurs, les prévisions de résultat sont aussi biaisées. Pour qualifier les prévisions (optimistes / pessimistes), nous avons repris la méthodologie de Eames et Glover [2003]. Le point de partage entre les prévisions optimistes et pessimistes (4) est donné par l'erreur commise au cours de l'année au sein de la population étudiée. Baik et al. [2009] mettent en évidence le fait que les analystes traitent différemment les titres dits " glamour» et ceux dits «value ». Les modifications de comportement en période de crise ont pu être différentes selon ces catégories de titres. Pour classer les entreprises, nous nous sommes référés au ratio market to book value (5) l'année qui précède l'étude. Enfin, on peut penser que les analystes concentrent leurs efforts sur des firmes qui enregistrent des mouvements communs de cours. Kini et al. [2009] observent que les courtiers non américains privilégient la spécialisation des analystes par pays. Si les effets de la crise se manifestent sur la synchronisation des révisions de prévisions et des variations de cours, alors des évolutions similaires doivent être observées au sein des principaux pays (6) concernés. 
A l'aide de ces 6 variables, nous avons créé six paires de sous-échantillons tirés au sein de l'échantillon de base. Concernant le nombre d'analystes, nous avons distingué les titres suivis par moins de 5 analystes (de 1 à 4 ) et ceux suivis par plus de 10 analystes (11 et plus). Les deux sous-échantillons représentent environ un tiers de l'effectif global. Les 2 variables, volume de transactions et mesure de BKLS ont été traitées de manière similaire. Chacune est estimée pour les 9 mois de la période retenue. Une moyenne est calculée par entreprise et par période. Les entreprises sont ensuite classées en ordre croissant. Le premier tiers constitue le sous-échantillon des entreprises à faible valeur, le dernier celui de celles à valeur élevée. Pour distinguer les firmes ( Value ») et celles ( Glamour »), nous avons classé par ordre croissant toutes les entreprises de l'échantillon en fonction du market to book ratio estimé en début d'année. Le premier tiers forme les firmes "Value », le dernier celles "Glamour ». La distinction entre les sociétés pour lesquelles les analystes étaient optimistes et celles pour lesquelles ils étaient pessimistes, est obtenue en calculant pour chaque firme la moyenne de l'erreur normée, puis en les classant par ordre croissant. La médiane départage les deux souséchantillons. Enfin, concernant la variable pays, nous Pour le facteur pays, nous distinguons simplement les entreprises allemandes et françaises car elles sont les plus nombreuses (environ un quart de l'échantillon pour chaque pays). Tous les classements ont été répétés chaque année.

$$
\text { <insérer le Tableau } 6>
$$

Au tableau 6, il apparaît que les révisions des prévisions de bénéfice avant la crise en 2006 sont positivement et significativement associées aux variations des cours boursiers des 3 mois écoulés. La relation est plus forte quand le nombre d'analystes est élevé, les volumes de transaction plus importants, l'asymétrie d'information plus faible, les prévisions plus pessimistes, les entreprises plus « Glamour», en France plus qu'en Allemagne. En revanche, les estimations ne font apparaître aucune relation positive et significative entre les variations de cours et les révisions de prévision qui précèdent. En 2008, la relation entre les révisions de prévision et les variations de cours boursiers persistent généralement. Deux différences apparaissent : le lien n'est plus significatif pour les titres peu suivis, il ne l'est plus non plus en Allemagne. Par ailleurs, les écarts entre les deux sous-populations sont plus marqués pour les volumes de transaction, le caractère optimiste ou pessimiste des prévisions. Ces résultats suggèrent que les analystes incorporent dans les prévisions qui sont publiées par I/B/E/S des informations qui sont déjà reflétées par le marché. Ce comportement ne disparaît pas et reste très marqué en période de crise pour les titres auxquels les analystes portent naturellement le plus d'intérêt (suivi plus grand et volumes de transaction élevés) ou pour lesquels les exigences pour les analystes sont les moins pesantes (asymétrie d'information plus faible, prévisions plus pessimistes).

\subsection{Fréquence de révision et effet des variations de cours sur l'intensité de la révision des prévisions de bénéfice par action}

En 2006, 38,1\% des observations (mois-firme) concernant les révisions de prévision de bénéfice ont des valeurs nulles : aucun analyste pour la firme en question n'avait modifié sa prévision au cours du mois concerné. En 2008 , ce pourcentage diminue à $28,4 \%$. La 
fréquence d'apparition d'un zéro est donc importante au sein de ces échantillons et susceptible d'affecter les estimations économétriques. Ce phénomène trouve son explication dans le cycle de suivi des firmes par les analystes. Les révisions sont fréquentes pour les entreprises de grande taille, plus rares pour les petites. Elles suivent souvent des annonces importantes, comme celle du dernier résultat connu et obéissent à un calendrier implicite. Nous abordons dans ce qui suit deux questions : (i) en période de crise, ce calendrier est-il modifié ? (ii) les révisions inhabituelles quant à leur positionnement dans le temps ont-elles un contenu informationnel différent? Pour répondre à ces deux interrogations, nous allons dans un premier temps estimer un modèle de prédiction d'occurrence d'une révision en fonction des données disponibles le mois précédent. Nous classons les firmes-mois en 2 catégories : révision attendue et révision non attendue en fonction de la probabilité estimée par le modèle probit. Si une révision n'est pas attendue pour un mois donné et une firme particulière, et que la révision a bien eu lieu, nous la considérons comme révision inhabituelle. Plus généralement, nous distinguons 3 situations : (1) la révision était attendue et se réalise, (2) la révision n'était pas attendue et se réalise, (3) la révision ne se réalise pas. Puis, nous estimons un modèle de régression avec la révision de la prévision de bénéfice par action comme variable dépendante et comme variable indépendante, la variation du cours boursier multipliée par une variable muette correspondant à l'une des 3 situations. Nous attendons un effet informationnel plus fort dans le cas où la révision est inhabituelle et une absence d'effet dans les cas où il n'y a pas de révision.

$<$ insérer le tableau $7>$

La partie A du tableau 7 fournit l'estimation d'un modèle probit où il apparaît que la fréquence est saisonnière. Bien que nous ayons restreint notre échantillon aux mois d'avril à janvier, il reste encore quelques entreprises (27 sur un total de 727) qui annoncent leurs résultats au cours de cette période. Le mois 0 est par convention celui de cette annonce. Une variable muette a été introduite pour chacun des autres mois. On peut constater que tous les coefficients sont négatifs : la probabilité d'une révision est la plus élevée en 0 et elle est la plus faible en 3, 10, 8 et 9 (coefficients négatifs et significatifs). Par ailleurs, l'importance de la couverture du titre par les analystes le mois précédent (mesurée ici par la variable Cov, logarithme du nombre d'analystes) affecte positivement et significativement la probabilité de révision, le phénomène étant encore plus marqué en 2008 (le coefficient associé à la variable composite, produit de Cov et d'une variable muette égale à 1 en 2008 et 0 en 2006, est positif et significatif). Enfin, la volatilité relative du titre le mois précédent $V o l$ (mesurée par le ratio de la variation mensuelle du cours boursier au carré et de la volatilité historique de l'action mesurée sur les 4 mois qui précèdent à partir de données quotidiennes) est elle aussi associée positivement. On peut noter cependant que l'effet de cette volatilité n'a pas été proportionnel en 2008 (le coefficient associé à la variable composite, produit de $\mathrm{Vol}$ et d'une variable muette égale à 1 en 2008 et 0 en 2006, est négatif et significatif). En résumé, les révisions sont plus fréquentes certains mois, pour les titres les plus suivis et après de fortes variations des cours.

La partie $\mathrm{B}$ du tableau 7 fournit l'estimation d'un modèle de régression où la variable dépendante est la révision de la prévision du bénéfice par action et les variables indépendantes 
sont les valeurs décalées des révisions (pour les 3 mois précédents), les variations des cours boursiers (pour le mois considéré et les 3 mois qui précèdent) et un ensemble de variables composites. Ces dernières sont obtenues en multipliant ces variations de cours par une combinaison de variables muettes, la première égale à 1 en 2008 (0 en 2006) et les autres représentant les situations (2) et (3). Les coefficients associés aux variables correspondant à des situations où il n'y a pas de révision sont négatifs. En effet, on s'attend à ce que la relation positive entre les variations de cours boursiers et les révisions de prévision n'apparaissent que quand il y a effectivement une révision. Les coefficients associés aux variations des cours boursiers sont significatifs, y compris pour les trois décalages $(0.032,0.026$ et 0.018$)$ quand la révision a lieu alors qu'elle était attendue. Ce résultat est amplifié en 2008 pour les trois valeurs décalées $(0.040,0.034$ et 0.045$)$. En revanche, l'association des valeurs contemporaines des deux variables n'est plus significative en 2008. Ces résultats confirment les précédents. L'introduction de la variable muette $D_{-} N P_{-} R$ permet de constater qu'en 2008, quand la révision se produit et qu'elle n'est pas attendue, son importance est liée aux fluctuations passées des cours avec une grande intensité pour un décalage d'un mois $(0.234)$ et de deux mois (0.077). Ce résultat suggère qu'en période de crise, les analystes révisent de manière inhabituelle leurs prévisions de bénéfices quand les cours ont fortement varié au cours des mois précédents. Le fait d'isoler ce phénomène dans la régression ne détruit pas les résultats plus généraux que nous avons précédemment mentionnés.

\section{Conclusion}

Cet article montre que, le contenu informationnel reflété par les variations de cours est pris en compte avec retard par le consensus des analystes européens et que le phénomène a été accentué lors de la crise de 2007-2008. L'association contemporaine entre les deux variables disparaît même en 2008. La relation entre les révisions de prévisions de bénéfice par action et les variations passées des cours boursiers est encore plus forte quand le nombre d'analystes est élevé et les prévisions plus précises. Ces résultats suggèrent que les analystes incorporent dans les prévisions publiées par I/B/E/S des informations déjà reflétées par le marché. En période de crise, ce comportement est encore plus marqué pour les titres intéressant le plus les analystes (suivi plus grand et volume de transactions élevé) ou pour lesquels les exigences pour les analystes sont les moins pesantes (asymétrie d'information plus faible, prévisions plus pessimistes). De plus, quand la révision se produit et qu'elle n'est pas attendue, son intensité est liée aux fluctuations passées des cours. Il apparaît ainsi qu'en période de crise, les analystes révisent de manière inhabituelle leurs prévisions de bénéfices quand les cours ont fortement varié au cours des mois précédents.

$\mathrm{Au}$ total, durant la crise financière de 2007-2008, l'information mise à disposition par les analystes à travers le consensus, suivait plus qu'elle ne précédait les mouvements des marchés. De ce point de vue, les analystes apparaissent plus comme des experts qui trient l'information, la valident et la rendent cohérente entre autre avec l'information comptable. Les prévisions de bénéfice par action procèdent moins d'un effort de recherche autonome que d'une tentative d'explicitation de fondamentaux soutenant les évaluations du marché. De ce point de vue, les analystes financiers jouent un rôle important pour aider les opérateurs à apprécier les évolutions des cours boursiers et les rendre intelligibles. Mais, les consensus publiés dans ces périodes agitées souffrent d'un plus grand retard dans les mises à jour. 


\section{Bibliographie :}

Ang, J. S., \& Ma, Y., (2001), The behavior of financial analysts during the Asian financial crisis in Indonesia, Korea, Malaysia, and Thailand, Pacific-Basin Finance Journal, 9, 233-263.

Bae, K-H.,Tan, H., \& Welker, M., (2008), International GAAP Differences: The Impact on Foreign Analysts. The Accounting Review, 83 (3), 593-628.

Baik, B., Farber, D. B., \& Petroni, K., (2009), Analysts' Incentives and Street Earnings. Journal of Accounting Research, 47(1).

Barber, B. M., Lehavy, R., \& Trueman, B., (2007), Comparing the Stock Recommendation Performance of Investment Banks and Independent Research Firms. Journal of Financial Economics, 85, 490-517.

Barron O., E., Byard, D., \& Kim, O., (2002), Changes in Analysts' Information around Earnings Announcements. The Accounting Review, 77 (4), 821-846.

Barron, O. E., Byard, D., \& Yu, Y. (2008). Earnings Surprises that Motivate Analysts to Reduce Average Forecast Error. The Accounting Review, 83 (2), 303-325.

Barron, O., Kim, O., Lim, S., \& Stevens, D., (1998), Using analyst forecasts to measure properties of analyst' information environment. The Accounting Review, 73(4), 421-434.

Barth, M. E., KaszniK, R., \& Mc Nichols, M. F. (2001). Analyst Coverage and Intangible Assets. Journal of Accounting Research, 39(1), 1-34.

Bartov, E., Givoly, D., \& Hayn, C. (2002). The rewards to meeting or beating earnings expectations. Journal of Accounting and Economics , 33, 173-204.

Basu, S., (1997), The conservatism principle and the asymmetric timeliness of earnings. Journal of Accounting \& Economics, 24 (1), 3-38.

Brown, C. O., (2009), Analyst Career Concerns and Asset Prices. Working paper, Zicklin School of Business, Baruch College, City University of New York, http://ssrn.com/abstract=1495138.

Brown, L., Richardson, G., \& Schwager, S.. (1987), An information interpretation of financial analyst superiority in forecasting earnings. Journal of Accounting Research, 25 (1), 1-49.

Bryan, D. M., \& Tiras, S. L., (2007), The Influence of Forecast Dispersion on the Incremental Explanatory Power of Earnings, Book Value, and Analyst Forecasts on Market Prices. The Accounting Review, 82(3), 651-677.

Chang Y. \& Chan, C, (2008), Financial analysts' stock recommendation revisions and stock price changes, Applied Financial economics, 18, 309-325

Chen, Q., \& Jiang, W., (2006), Analysts' Weighting of Private and Public Information. The Review of Financial Studies, 19 (1).

Clement M. B., \& Tse S. Y., (2003), Do Investors Respond to Analysts' Forecast Revisions as if Forecast Accuracy Is All That Matters? The Accounting Review, 78 (1), 227-249.

Eames, M. J., \& Glover, S. M., (2003), Earnings Predictability and the Direction of Analysts' Earnings Forecast Errors. The Accounting Review, 78 (3), 707-724.

Frankel, R. M., Kothari, S. P., \& Weber, J., (2006). Determinants of the informativeness of analyst research. Journal of Accounting and Economics, 41, 29-54.

Freeman, R., \& Tse, S., (1992), A nonlinear model of security price responses to unexpected earnings. Journal of Accounting Research, 30 (2), 185-209.

Givoly, D., Hayn, C., \& Lehavy, R., (2009), The Quality of Analysts' Cash Flow Forecasts. The Accounting Review, 84 (6), 1877-1911.

Givoly, D., \& Lakonishok J., (1979), The information content of financial analysts' forecasts of earnings. Journal of Accounting and Economics, 1, 165-185. 
Givoly, D., \& Lakonishok J., (1980), Financial analysts' forecast of earnings: The value to investors. Journal of Banking and Finance, 4, 221-233.

Gleason, C. A., \& Lee, C. M., (2003), Analyst Forecast Revisions and Market Price Discovery. The Accounting Review, 78 (1), 193-225.

Greene, W. H., (2000), Econometric Analysis, Prentice-Hall, $4^{\text {th }}$ Edition.

Green T.C., (2006), The value of client access to analyst recommendations, Journal of Financial and Quantitative Analysis, 41, p. 1-24.

Griffin, P., (1976), Competitive information in the stock market: An empirical study of earnings, dividends, and analysts' forecasts. Journal of Finance, 31, 631-650.

Hurlin, C., (2005), "Un test simple de l'hypothèse de non-causalité dans un modèle de panel hétérogène." Revue Economique 56 (3), 799-809.

Imhoff, E., \& Lobo, G., (1984), Information content of analysts' composite forecast revisions. Journal of Accounting Research, 22 (Autumn), 541-554.

Kini, O., Mian, S., Rebello, M., \& Venkateswaran, A., (2009), On the Structure of Analyst Research Portfolios and Forecast Accuracy. Journal of Accounting Research, 47 (4).

Kothari, S. P., \& Kolasinski, A. C. (2008). Investment Banking and Analyst Objectivity: Evidence from Analysts Affiliated with Mergers and Acquisitions Advisors. Journal of Financial and Quantitative Analysis, 43(4), 817-842.

Kothari, S. P., Li, X., \& Short, J. E., (2009) The Effect of Disclosures by Management, Analysts, and Business Press on Cost of Capital, Return Volatility, and Analyst Forecasts: A Study Using Content Analysis. The Accounting Review, 84(5), 1639-1670.

Lang, M., \& Lundholm, R., (1996) Corporate Disclosure Policy and Analyst Behavior, The Accounting Review, (October), 467-92.

Lin, H., \& McNichols, M., (1998), Underwriting relationships, analysts' earnings forecasts and investment recommendations. Journal of Accounting and Economics, 25, 101-127.

Ljungqvist, A., Marston, F., Starks, L. T., Wei, K. D., \& Yan, H., (2007), Conflicts of interest in sellside research and the moderating role of institutional investors. Journal of Financial Economics, $85,420-456$.

Michaely, R., \& Womack, K., (1999), Conflict of interest and the credibility of underwriter analyst recommendations. Review of Financial Studies, 12, 573-608.

Mikhail, M., Walther, B., \& Willis, R., (1999), Does forecasting accuracy matter to security analysts? The Accounting Review, 74 (2), 185-200.

Miller, G., (2002), Earnings performance and discretionary disclosure. Journal of Accounting Research, 40 (1), 173-204.

Petersen, M., (2009), Estimating standard errors in finance panel data sets: Comparing approaches, Review of Financial Studies, 22, 435-480.

Sedor, L. M., (2002), An Explanation for Unintentional Optimism in Analysts' Earnings Forecasts. The Accounting Review Vol. 77 (4), 731-753.

Schipper, K., (1991), Analysts' forecasts. Accounting Horizons, 54 (December), 105-122.

Stickel, S. (1989). The timing of and incentives for annual earnings forecasts near interim financial report releases. Journal of Accounting and Economics, 11 (2-3), 275-92.

Thompson, S. B., (2009), Simple formulas for standard errors that cluster by both firm and time, Working Paper, available at: http://ssrn.com/abstract $=914002$.

Waymire, G., (1985), Earnings volatility and voluntary management forecast disclosure. Journal of Accounting Research, 23 (1), 268-269. 
Wiedman, C., (1996), The relevance of characteristics of the information environment in the selection of a proxy for the market's expectations for earnings: An extension of Brown, Richardson, and Schwager (1987). Journal of Accounting Research, 34(2), 313-324. 


\section{Tableau 1}

\section{Constitution de l'échantillon}

L'échantillon contient des entreprises non financières de la zone Euro sur la période 2006-2008. Les prévisions et les réalisations des bénéfices par action proviennent de la base I/B/E/S de Thomson Financials, de même que les recommandations des analystes. Les cours boursiers et les autres données financières ont été obtenus auprès de la base TF de Thomson Financials. Ce tableau décrit le processus de sélection de l'échantillon.

\begin{tabular}{|c|c|c|c|c|c|c|c|c|c|}
\hline & 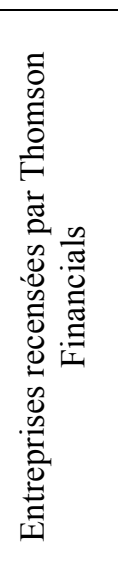 & 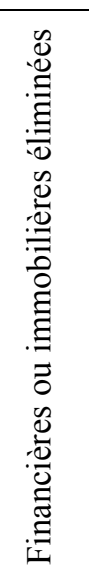 & 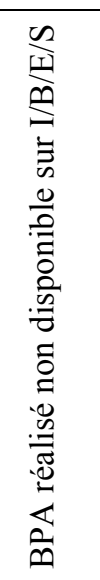 & 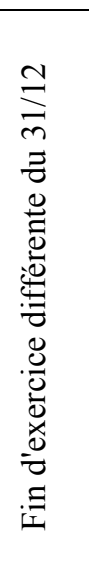 & 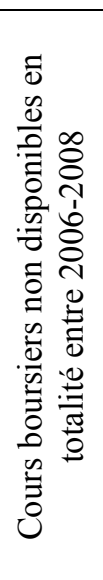 & 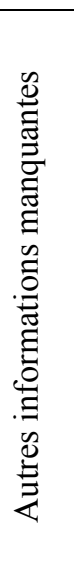 & 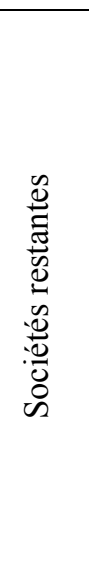 & 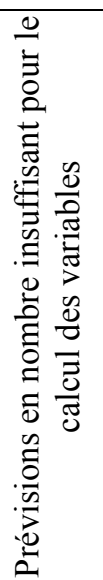 & 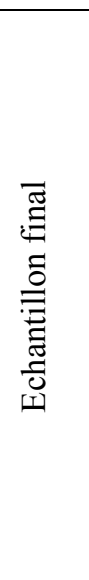 \\
\hline Allemagne & 1468 & 369 & 535 & 87 & 58 & 41 & 378 & 221 & 157 \\
\hline Autriche & 190 & 48 & 79 & 23 & 3 & 3 & 34 & 16 & 18 \\
\hline Belgique & 290 & 90 & 113 & 7 & 13 & 5 & 62 & 17 & 45 \\
\hline Chypre & 1 & 0 & 1 & 0 & 0 & 0 & 0 & 0 & 0 \\
\hline Espagne & 299 & 91 & 101 & 6 & 13 & 0 & 88 & 37 & 51 \\
\hline Finlande & 222 & 36 & 72 & 6 & 8 & 2 & 98 & 23 & 75 \\
\hline France & 1584 & 329 & 768 & 125 & 49 & 17 & 296 & 135 & 161 \\
\hline Grèce & 416 & 71 & 90 & 11 & 25 & 17 & 202 & 170 & 32 \\
\hline Irlande & 151 & 34 & 49 & 27 & 15 & 3 & 23 & 10 & 13 \\
\hline Italie & 498 & 124 & 145 & 13 & 36 & 5 & 175 & 95 & 80 \\
\hline Luxembourg & 64 & 26 & 18 & 3 & 2 & 0 & 15 & 7 & 8 \\
\hline Pays-Bas & 391 & 90 & 161 & 12 & 21 & 1 & 106 & 33 & 73 \\
\hline Portugal & 127 & 29 & 68 & 0 & 3 & 1 & 26 & 12 & 14 \\
\hline Slovaquie & 34 & 5 & 28 & 0 & 0 & 0 & 1 & 1 & 0 \\
\hline Slovénie & 13 & 1 & 5 & 0 & 0 & 0 & 7 & 7 & 0 \\
\hline Total & 5748 & 1343 & 2233 & 320 & 246 & 95 & 1511 & 784 & 727 \\
\hline
\end{tabular}




\section{Tableau 2}

\section{Présentation de l'échantillon}

Ce tableau reporte les statistiques descriptives de synthèse sur les variables utilisées dans cette étude : prévision de bénéfice par action pour l'exercice suivant et révision de la prévision. Ces variables sont observées à une fréquence mensuelle. Les taux de révision des prévisions de bénéfice est calculé pour chaque entreprise et chaque mois en divisant le nombre des révisions effectués par les nombres de prévisions. Les informations données sont des moyennes pour la période allant de mai 2006 (2008) à janvier 2007 (2009). Les capitalisations boursières sont celles fournies par Thomson Financials au 31 décembre de l'année en millions d'euros.

Panel A : Ventilation par décile de taille/année (selon la capitalisation boursière) :

\begin{tabular}{|c|c|c|c|c|c|c|c|c|c|}
\hline & Nombre & \multicolumn{4}{|c|}{ Capitalisation boursière } & \multicolumn{4}{|c|}{ Prévisions de bénéfice par action } \\
\hline & $\begin{array}{c}\mathrm{de} \\
\text { sociétés }\end{array}$ & \multicolumn{2}{|c|}{$\begin{array}{c}\text { Moyenne } \\
\text { M€ }\end{array}$} & \multicolumn{2}{|c|}{$\begin{array}{l}\text { Médiane } \\
\text { M€ }\end{array}$} & \multicolumn{2}{|c|}{$\begin{array}{l}\text { Nombre } \\
\text { Moyenne }\end{array}$} & \multicolumn{2}{|c|}{$\begin{array}{l}\% \text { de révisions par } \\
\text { rapport au nombre }\end{array}$} \\
\hline Déciles & & 2006 & 2008 & 2006 & 2008 & 2006 & 2008 & 2006 & 2008 \\
\hline 1 & 72 & 47 & 21 & 47 & 23 & 2.2 & 2.3 & $22 \%$ & $26 \%$ \\
\hline 2 & 73 & 129 & 50 & 130 & 49 & 3.6 & 3.3 & $25 \%$ & $29 \%$ \\
\hline 3 & 73 & 234 & 98 & 226 & 101 & 4.7 & 4.6 & $25 \%$ & $31 \%$ \\
\hline 4 & 73 & 383 & 166 & 388 & 162 & 6.1 & 6.4 & $26 \%$ & $33 \%$ \\
\hline 5 & 73 & 603 & 289 & 604 & 292 & 7.1 & 8.6 & $30 \%$ & $33 \%$ \\
\hline 6 & 73 & 998 & 471 & 986 & 466 & 7.7 & 9.6 & $27 \%$ & $36 \%$ \\
\hline 7 & 73 & 1758 & 804 & 1743 & 797 & 10.5 & 10.8 & $30 \%$ & $34 \%$ \\
\hline 8 & 73 & 3166 & 1610 & 3088 & 1577 & 12.8 & 14.3 & $28 \%$ & $34 \%$ \\
\hline 9 & 72 & 7257 & 3930 & 6812 & 3859 & 17.6 & 17.6 & $30 \%$ & $38 \%$ \\
\hline 10 & 72 & 31289 & 22246 & 21291 & 12740 & 24.4 & 25.4 & $32 \%$ & $35 \%$ \\
\hline Total & 727 & 4552 & 2945 & 717 & 356 & 9.6 & 10.3 & $27 \% \%^{(1)}$ & $33 \%^{(1)}$ \\
\hline
\end{tabular}

(1) différence significative au seuil de $1 \%$ 
Panel B : Ventilation par pays :

\begin{tabular}{|c|c|c|c|c|c|c|c|c|c|}
\hline & \multirow{2}{*}{$\begin{array}{l}\text { Nombre } \\
\text { de } \\
\text { sociétés }\end{array}$} & \multicolumn{4}{|c|}{ Capitalisation boursière } & \multicolumn{4}{|c|}{ Prévisions de bénéfice par action } \\
\hline & & \multicolumn{2}{|c|}{ Moyenne } & \multicolumn{2}{|c|}{ Somme } & \multicolumn{2}{|c|}{$\begin{array}{l}\text { Nombre } \\
\text { Moyenne }\end{array}$} & \multicolumn{2}{|c|}{$\begin{array}{l}\% \text { de révisions par } \\
\text { rapport au nombre }\end{array}$} \\
\hline Pays & & 2006 & 2008 & 2006 & 2008 & 2006 & 2008 & 2006 & 2008 \\
\hline Allemagne & 157 & 4520 & 3501 & 709616 & 549647 & 10.4 & 11.6 & $23 \%$ & $29 \%$ \\
\hline Autriche & 18 & 2667 & 1487 & 48014 & 26773 & 6.3 & 8.7 & $27 \%$ & $34 \%$ \\
\hline Belgique & 45 & 1604 & 969 & 72196 & 43626 & 6.0 & 6.3 & $30 \%$ & $34 \%$ \\
\hline Espagne & 51 & 6870 & 4932 & 350362 & 251525 & 13.8 & 15.2 & $21 \%$ & $24 \%$ \\
\hline Finlande & 75 & 2373 & 1291 & 177997 & 96848 & 9.3 & 9.0 & $36 \%$ & $41 \%$ \\
\hline France & 161 & 5762 & 3582 & 927605 & 576709 & 9.9 & 10.0 & $32 \%$ & $36 \%$ \\
\hline Grèce & 32 & 1809 & 964 & 57887 & 30836 & 8.0 & 8.8 & $22 \%$ & $27 \%$ \\
\hline Irlande & 13 & 2296 & 1084 & 29843 & 14090 & 4.6 & 5.0 & $18 \%$ & $27 \%$ \\
\hline Italie & 80 & 4797 & 2600 & 383749 & 207994 & 9.2 & 10.1 & $21 \%$ & $32 \%$ \\
\hline Luxembourg & 8 & 6090 & 3590 & 48722 & 28724 & 5.6 & 7.9 & $31 \%$ & $33 \%$ \\
\hline Pays-Bas & 73 & 6231 & 3928 & 454878 & 286770 & 10.9 & 10.9 & $31 \%$ & $35 \%$ \\
\hline Portugal & 14 & 3467 & 1946 & 48541 & 27237 & 8.0 & 9.5 & $21 \%$ & $28 \%$ \\
\hline
\end{tabular}




\section{Tableau 3}

\section{Présentation des prévisions de bénéfice par action}

Ce tableau reporte les statistiques descriptives sur la qualité des prévisions de bénéfice par action prises en compte et sur les révisions des prévisions des bénéfices par action. Le nombre d'analystes financiers est le nombre moyen des analystes suivant les sociétés en 2006 et 2008, pour les exercices 2007 et 2009 . Les résultats ont été obtenus sur un échantillon de 727 entreprises de la zone Euro. Au Panel A, la précision est mesurée par la moyenne des erreurs absolues. Le biais est mesuré par la moyenne des erreurs. L'âge de la prévision est exprimé en mois et mesuré par rapport à la date d'annonce du bénéfice. Au Panel B, nous observons les révisions. Les mois de mai, septembre et janvier correspondent au début, au milieu et à la fin de notre période d'observation.

\section{Panel A : Qualité des prévisions de bénéfice par action}

\begin{tabular}{|c|c|c|c|c|c|c|c|c|}
\hline & \multicolumn{4}{|c|}{ Précision } & \multicolumn{4}{|c|}{ Biais } \\
\hline & \multicolumn{2}{|c|}{2006} & \multicolumn{2}{|c|}{2008} & \multicolumn{2}{|c|}{2006} & \multicolumn{2}{|c|}{2008} \\
\hline Age de la prévision (en mois) & 20 & 14 & 20 & 14 & 20 & 14 & 20 & 14 \\
\hline Nombre d'analystes & & & & & & & & \\
\hline$\{1-5\{$ & $4.56 \%$ & $4.29 \%$ & $8.89 \%$ & $7.00 \%$ & $-0.55 \%$ & $-0.08 \%$ & $-6.72 \%$ & $-4.07 \%$ \\
\hline$\{5-10\}$ & $3.02 \%$ & $2.43 \%$ & $7.70 \%$ & $4.97 \%$ & $-0.62 \%$ & $-0.45 \%$ & $-6.78 \%$ & $-3.44 \%$ \\
\hline$\{10-49\}$ & $2.24 \%$ & $1.77 \%$ & $5.68 \%$ & $3.53 \%$ & $-0.15 \%$ & $-0.10 \%$ & $-5.40 \%$ & $-2.95 \%$ \\
\hline Total & $3.31 \%{ }^{(2)}$ & $2.86 \% \%^{(2)}$ & $7.07 \% \%^{(1),(2)}$ & $4.77 \%^{(1),(2)}$ & $-0.43 \% \%^{(2)}$ & $-0.20 \% \%^{(2)}$ & $-6.12 \%{ }^{(1),(2)}$ & $-3.36 \% \%^{(1),(2)}$ \\
\hline
\end{tabular}

(1) : différence significative à $1 \%$ entre des âges différents (20 ou 14) au sein de la même année, (2) : différence significative à $1 \%$ entre 2 années (2006 et 2008 ), au même âge (20 ou 14),

\section{Panel B : Révision des prévisions de bénéfice par action}

\begin{tabular}{|c|c|c|c|c|c|c|c|c|c|c|c|c|}
\hline & \multicolumn{12}{|c|}{ Prévisions de bénéfice par action } \\
\hline & \multicolumn{6}{|c|}{ Nombre (moyenne) } & \multicolumn{6}{|c|}{$\%$ de révisions par rapport au nombre } \\
\hline & \multicolumn{3}{|c|}{ Exercice 2007} & \multicolumn{3}{|c|}{ Exercice 2009} & \multicolumn{3}{|c|}{ Exercice 2007} & \multicolumn{3}{|c|}{ Exercice 2009} \\
\hline Mois de la prévision & mai 2006 & sept 2006 & janv 2007 & mai 2008 & sept 2008 & janv 2009 & mai 2006 & sept 2006 & janv 2007 & mai 2008 & sept 2008 & janv 2009 \\
\hline Nombre d'analystes & & & & & & & & & & & & \\
\hline$[1-5[$ & 2,6 & 2,9 & 3,2 & 2,8 & 2,7 & 2,6 & $23,50 \%$ & $30,98 \%$ & $27,03 \%$ & $22,82 \%$ & $23,49 \%$ & $38,58 \%$ \\
\hline$[5-10]$ & 6,5 & 7,4 & 8,1 & 7,2 & 7,6 & 7,0 & $24,16 \%$ & $29,72 \%$ & $31,57 \%$ & $28,72 \%$ & $23,22 \%$ & $52,23 \%$ \\
\hline ] $10-49$ ] & 16,8 & 18,6 & 19,0 & 17,6 & 18,5 & 18,4 & $24,03 \%$ & $30,47 \%$ & $39,78 \%$ & $28,07 \%$ & $28,65 \%$ & $52,13 \%$ \\
\hline Total & 8,7 & 9,7 & 10,2 & 10,0 & 10,4 & 10,2 & $23,88 \%$ & $30,44 \% \%^{(1)}$ & $32,82 \%^{(1)}$ & $26,45 \%$ & $25,53 \%{ }^{(1)}$ & $47,55 \% \%^{(1)}$ \\
\hline
\end{tabular}

(1) différence significative au seuil de $1 \%$ entre deux années différentes, à la même époque (septembre ou janvier) 
Tableau 4

Test de causalité de Granger - Variation des cours boursiers et révision des prévisions de bénéfice par action effectuées par les analystes.

Le tableau expose les résultats obtenus sur un échantillon de 727 entreprises de la zone Euro sur la période 2006-2008. $R$ est la variation des cours boursiers au cours d'un mois en tenant compte des dividendes et des opérations sur le capital. VE est la variation du consensus moyen des bénéfices par action au cours du mois, normée par le cours de l'action au début 2006. Des effets fixes par firme et période (temps mesuré par rapport au mois de publication des résultats) ont été inclus mais ne sont pas rapportés. Les $T$ sont calculés à partir d'écart-types estimés avec des clusters par firme et par période

Panel A - Variable dépendante : $R_{i, m}$

\begin{tabular}{|c|c|c|c|c|c|c|c|c|}
\hline & \multicolumn{4}{|c|}{2006} & \multicolumn{4}{|c|}{2008} \\
\hline & \multicolumn{2}{|c|}{ Modèle réduit } & \multicolumn{2}{|c|}{ Modèle complet } & \multicolumn{2}{|c|}{ Modèle réduit } & \multicolumn{2}{|c|}{ Modèle complet } \\
\hline & coef & Clust. T & coef & Clust. T & coef & Clust. T & coef & Clust. T \\
\hline \multicolumn{9}{|c|}{ Variation des cours boursiers } \\
\hline$R_{i, m-1}$ & -0.185 & -8.443 & -0.184 & -8.614 & -0.215 & -9.041 & -0.215 & -9.045 \\
\hline$R_{i, m-2}$ & -0.122 & -6.327 & -0.121 & -5.989 & -0.270 & -16.169 & -0.268 & -16.891 \\
\hline$R_{i, m-3}$ & -0.088 & -5.690 & -0.085 & -5.507 & -0.135 & -8.170 & -0.133 & -7.903 \\
\hline \multicolumn{9}{|c|}{ Variation du bénéfice par action moyen prévu } \\
\hline$V E_{i, m}$ & 0.252 & 3.928 & 0.192 & 2.231 & -0.054 & -0.952 & -0.095 & -1.515 \\
\hline$V E_{i, m-1}$ & & & -0.130 & -2.436 & & & -0.098 & -2.277 \\
\hline$V E_{i, m-2}$ & & & -0.059 & -1.461 & & & -0.047 & -0.884 \\
\hline$V E_{i, m-3}$ & & & -0.112 & -0.971 & & & -0.081 & -1.455 \\
\hline Nbre d'Obs. & 6543 & & 6543 & & 6543 & & 6543 & \\
\hline Stat. de Fisher & 1.931 & $\begin{array}{l}p \text { value } \\
(0.000)\end{array}$ & 1.930 & $\begin{array}{l}p \text { value } \\
(0.000)\end{array}$ & 2.560 & $\begin{array}{l}p \text { value } \\
(0.000)\end{array}$ & 2.581 & $\begin{array}{l}p \text { value } \\
(0.000)\end{array}$ \\
\hline $\mathrm{R}^{2}$ ajusté & $9.6 \%$ & & $9.6 \%$ & & $15.0 \%$ & & $15.3 \%$ & \\
\hline \multicolumn{9}{|c|}{ Test de causalité de Granger } \\
\hline $\mathrm{S}$ & 1.631 & $\mathrm{~F}$ & 0.180 & & 7.023 & $\mathrm{~F}$ & 0.000 & \\
\hline
\end{tabular}

Panel B - Variable dépendante : $V E_{i, m}$

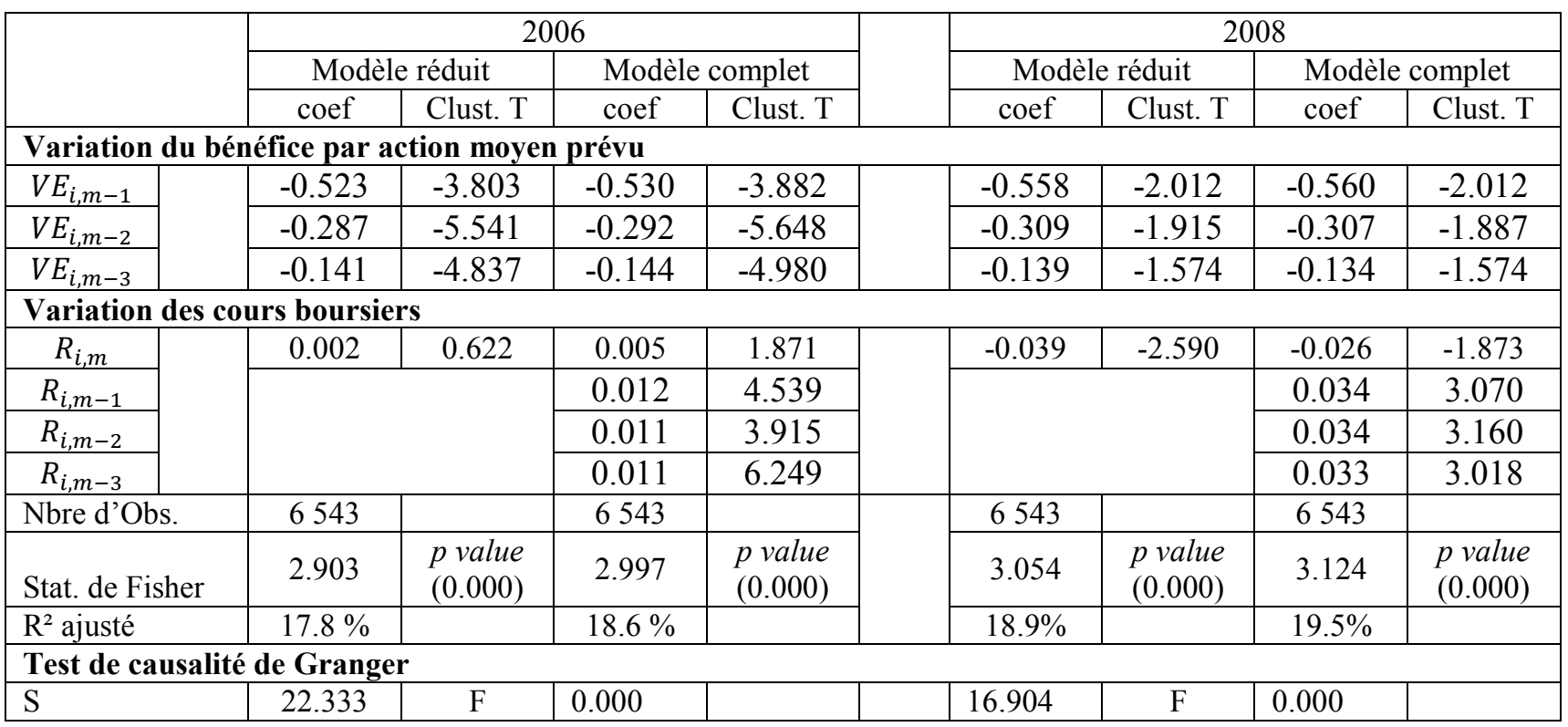


Tableau 5

Estimations des différences dans les effets des variables indépendantes entre 2008 et 2006

Le tableau expose les résultats obtenus sur un échantillon de 727 entreprises de la zone Euro sur la période 2006-2008. $R$ est la variation des cours boursiers au cours d'un mois en tenant compte des dividendes et des opérations sur le capital. DR est la variation des cours boursiers au cours d'un mois en tenant compte des dividendes et des opérations sur le capital multipliée par une variable muette égale à 1 si l'année est 2008, 0 sinon. VE est la variation du consensus moyen des bénéfices par action au cours du mois, normée par le cours de l'action au début 2006. DVE est la variation du consensus moyen des bénéfices par action au cours du mois, normée par le cours de l'action au début 2006 multipliée par une variable muette égale à 1 si l'année est 2008, 0 sinon. Des effets fixes par firme et période (temps mesuré par rapport au mois de publication des résultats) ont été inclus mais ne sont pas rapportés. Les T sont calculés à partir d'écart-types estimés avec des clusters par firme et par période

Variation des cours boursiers et révision des prévisions de bénéfice par action effectuées par les analystes

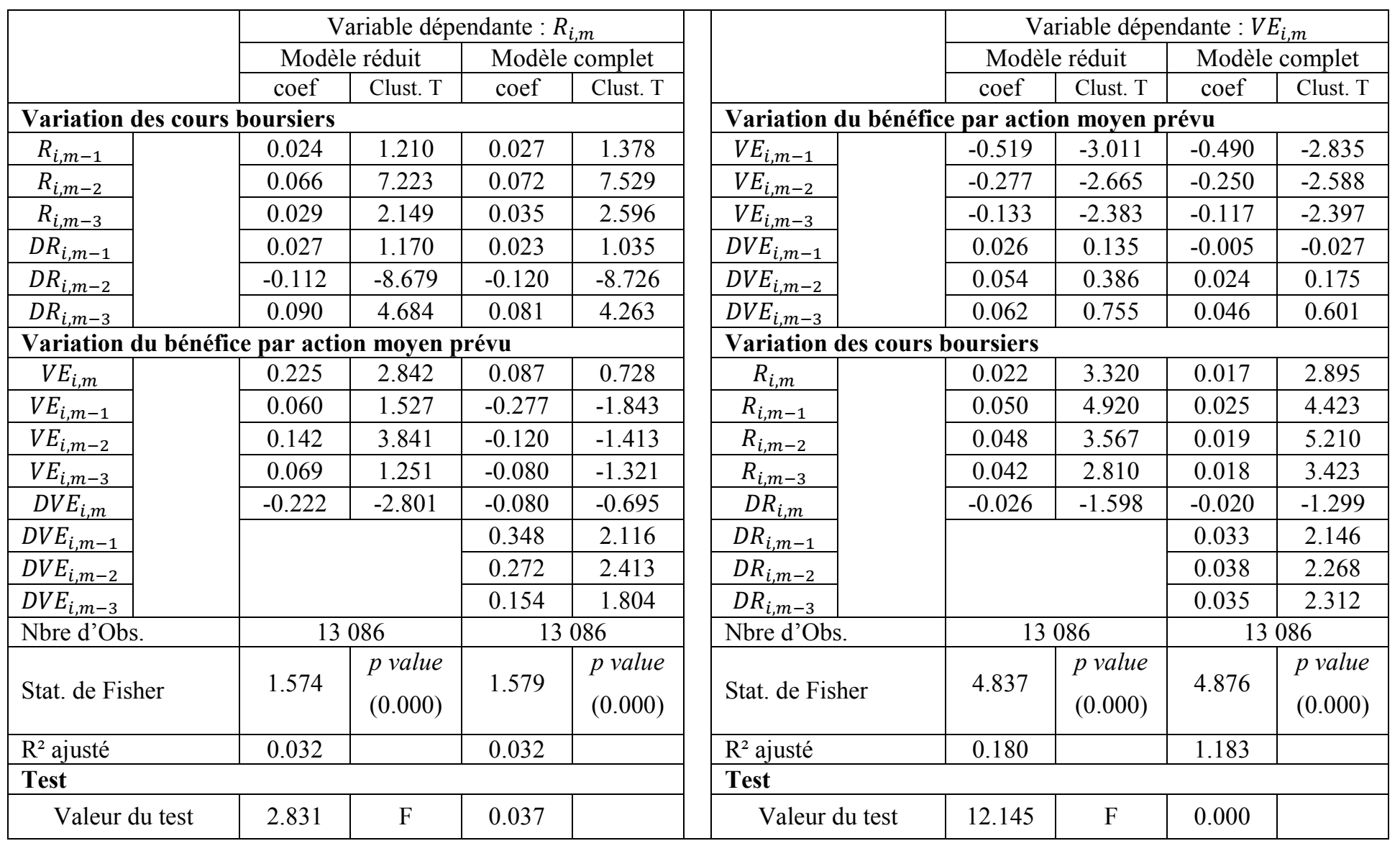




\section{Tableau 6}

\section{Etudes par sous-échantillons}

Le tableau expose les résultats obtenus sur des échantillons d'entreprises de la zone Euro sur la période 20062008. VE est la variation du consensus moyen des bénéfices par action au cours du mois, normée par le cours de l'action au début 2006. R est la variation des cours boursiers au cours d'un mois en tenant compte des dividendes et des opérations sur le capital. Des effets fixes par firme et période (temps mesuré par rapport au mois de publication des résultats) ont été inclus. Par parcimonie, seuls les tests de causalité de Granger et une indication sur le nombre des coefficients présents dans le modèle complet et absents du modèle réduit qui sont positifs et significatifs, sont repris dans ce tableau.

\begin{tabular}{|c|c|c|c|c|c|c|c|c|c|}
\hline \multirow{3}{*}{$\begin{array}{l}\text { Clé de } \\
\text { répartition }\end{array}$} & \multirow{3}{*}{$\begin{array}{c}\text { Sous } \\
\text { échantillon }\end{array}$} & \multirow{3}{*}{$\begin{array}{l}\text { Variable } \\
\text { dépen- } \\
\text { dante }\end{array}$} & \multirow{3}{*}{$\begin{array}{c}\text { Variable } \\
\text { indépen- } \\
\text { dante }\end{array}$} & \multicolumn{3}{|c|}{2006} & \multicolumn{3}{|c|}{2008} \\
\hline & & & & \multicolumn{2}{|c|}{ Test de Granger } & \multirow{2}{*}{$\begin{array}{l}\text { Coefficients } \\
\text { Sign }\end{array}$} & \multicolumn{2}{|c|}{ Test de Granger } & \multirow{2}{*}{$\begin{array}{l}\text { Coefficients } \\
\text { Sign }\end{array}$} \\
\hline & & & & $\mathrm{S}$ & $\mathrm{F}$ & & $\mathrm{S}$ & $\mathrm{F}$ & \\
\hline \multirow{4}{*}{ 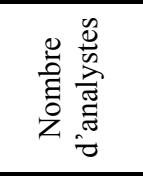 } & $\mathrm{N}>10$ & $\mathrm{R}$ & $\mathrm{VE}$ & 2.351 & 0.071 & 0 & 7.822 & 0.000 & 0 \\
\hline & $\mathrm{N}<5$ & $\mathrm{R}$ & VE & 0.759 & 0.517 & 0 & 5.376 & 0.001 & 0 \\
\hline & $\mathrm{N}>10$ & $\mathrm{VE}$ & $\mathrm{R}$ & 29.968 & 0.000 & 3 & 71.259 & 0.000 & 3 \\
\hline & $\mathrm{N}<5$ & $\mathrm{VE}$ & $\mathrm{R}$ & 4.323 & 0.005 & 3 & 0.676 & 0.567 & 0 \\
\hline \multirow{4}{*}{ 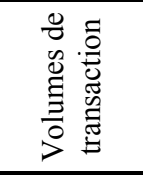 } & Indice $>2 / 3$ & $\mathrm{R}$ & $\mathrm{VE}$ & 5.130 & 0.002 & 0 & 17.475 & 0.000 & 0 \\
\hline & Indice $<1 / 3$ & $\mathrm{R}$ & $\mathrm{VE}$ & 0.725 & 0.537 & 0 & 3.503 & 0.015 & 0 \\
\hline & Indice $>2 / 3$ & $\mathrm{VE}$ & $\mathrm{R}$ & 34.294 & 0.000 & 3 & 45.723 & 0.000 & 2 \\
\hline & Indice $<1 / 3$ & $\mathrm{VE}$ & $\mathrm{R}$ & 3.594 & 0.013 & 2 & 3.509 & 0.015 & 3 \\
\hline \multirow{4}{*}{ 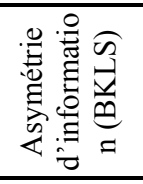 } & Indice $>2 / 3$ & $\mathrm{R}$ & $\mathrm{VE}$ & 2.168 & 0.090 & 0 & 1.189 & 0.312 & 0 \\
\hline & Indice $<1 / 3$ & $\mathrm{R}$ & $\mathrm{VE}$ & 3.755 & 0.011 & 0 & 7.879 & 0.000 & 0 \\
\hline & Indice $>2 / 3$ & $\mathrm{VE}$ & $\mathrm{R}$ & 12.901 & 0.000 & 3 & 15.896 & 0.000 & 2 \\
\hline & Indice $<1 / 3$ & $\mathrm{VE}$ & $\mathrm{R}$ & 30.456 & 0.000 & 3 & 24.870 & 0.000 & 2 \\
\hline \multirow{4}{*}{ 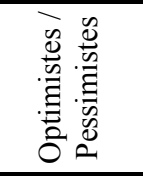 } & Indice $>1 / 2$ & $\mathrm{R}$ & $\mathrm{VE}$ & 1.663 & 0.173 & 0 & 3.986 & 0.008 & 0 \\
\hline & Indice $<1 / 2$ & $\mathrm{R}$ & $\mathrm{VE}$ & 2.259 & 0.080 & 0 & 5.169 & 0.001 & 0 \\
\hline & Indice $>1 / 2$ & $\mathrm{VE}$ & $\mathrm{R}$ & 15.478 & 0.000 & 3 & 12.549 & 0.000 & 2 \\
\hline & Indice $<1 / 2$ & $\mathrm{VE}$ & $\mathrm{R}$ & 28.435 & 0.000 & 3 & 61.483 & 0.000 & 2 \\
\hline \multirow{4}{*}{ 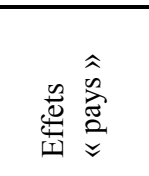 } & Allemagne & $\mathrm{R}$ & $\mathrm{VE}$ & 0.159 & 0.925 & 0 & 5.198 & 0.001 & 0 \\
\hline & France & $\mathrm{R}$ & $\mathrm{VE}$ & 4.755 & 0.003 & 0 & 1.122 & 0.339 & 0 \\
\hline & Allemagne & $\mathrm{VE}$ & $\mathrm{R}$ & 3.629 & 0.013 & 2 & 0.277 & 0.842 & 0 \\
\hline & France & $\mathrm{VE}$ & $\mathrm{R}$ & 6.394 & 0.000 & 2 & 27.166 & 0.000 & 2 \\
\hline
\end{tabular}


Tableau 7

Fréquence de révision et effet des variations de cours sur l’intensité de la révision des prévisions de bénéfice par action

Le tableau expose les résultats obtenus sur un échantillon de 727 entreprises de la zone Euro sur la période 2006-2008. Dans la partie A, les variables Mois_01 à Mois_11 sont des variables muettes qui prennent la valeur 1 si le mois considéré se situe de 1 à 11 mois après le mois e publication du dernier résultat, 0 sinon. La variable Cov est égale au logarithme du nombre d'analystes suivant le titre le mois qui précède. La variable Vol est égale au ratio, mesuré le mois précédent, de la variation mensuelle du cours boursier au carré et de la volatilité historique de l'action calculée à partir de données quotidiennes relevées sur 4 mois. D est une variable muette égale à 1 si l'année est 2008, 0 sinon. Elle multiplie les variables Cov et Vol. Dans la partie B, R est la variation des cours boursiers au cours d'un mois en tenant compte des dividendes et des opérations sur le capital. VE est la variation du consensus moyen des bénéfices par action au cours du mois, normée par le cours de l'action au début 2006. $D \_N P \_R$ est une variable muette égale à 1 si la révision de la prévision du bénéfice par action a eu lieu alors qu'elle n'est pas attendue suivant le modèle probit estimé dans la partie A, 0 sinon. D_NR est une variable muette correspondant aux cas où la révision n'a pas été réalisée. Des effets fixes par firme et période (temps mesuré par rapport au mois de publication des résultats) ont été inclus mais ne sont pas rapportés. Les $T$ sont calculés à partir d'écart-types estimés avec des clusters par firme et par période.

Table A : Modèle Probit d'estimation de la probabilité d'occurrence d'une révision de la prévision du bénéfice par action au sein du consensus

\begin{tabular}{|c|c|c|c|}
\hline \multicolumn{4}{|c|}{ Variable dépendante : 1 si il y a une révision du consensus, 0 sinon } \\
\hline Variables & Coefficients & t-value & $\mathrm{p}>|\mathrm{t}|$ \\
\hline Constante & -0.104 & -0.54 & 0.592 \\
\hline Mois_01 & -0.028 & -0.14 & 0.892 \\
\hline Mois_02 & -0.372 & -1.89 & 0.059 \\
\hline Mois_03 & -0.521 & -2.65 & 0.008 \\
\hline Mois_04 & -0.231 & -1.18 & 0.240 \\
\hline Mois_05 & -0.233 & -1.18 & 0.236 \\
\hline Mois_06 & -0.363 & -1.85 & 0.065 \\
\hline Mois_07 & -0.280 & -1.42 & 0.155 \\
\hline Mois_08 & -0.459 & -2.33 & 0.020 \\
\hline Mois_09 & -0.436 & -2.21 & 0.027 \\
\hline Mois_10 & -0.494 & -2.49 & 0.013 \\
\hline Mois 11 & -0.268 & -1.21 & 0.228 \\
\hline Cov & 0.395 & 27.21 & 0.000 \\
\hline DCov & 0.148 & 11.70 & 0.000 \\
\hline Vol & 1.704 & 4.36 & 0.000 \\
\hline DVol & -0.860 & -2.07 & 0.038 \\
\hline \multicolumn{3}{|c|}{ Nombre d'observations } & 13086 \\
\hline \multicolumn{3}{|c|}{ Likelihood Ratio Chi-square } & 1693.802 \\
\hline \multicolumn{3}{|c|}{ avec 15 degrés de liberté., prob $=0.000$} & \\
\hline \multicolumn{3}{|c|}{ Pourcentage de prédictions correctes } & $71.07 \%$ \\
\hline
\end{tabular}


Table B : Modèle de régression entre les révisions de prévision de bénéfice par action et les variations du cours boursier

\begin{tabular}{|c|c|c|c|c|c|c|c|}
\hline \multicolumn{8}{|c|}{ Variable dépendante $: V E_{i, m}$} \\
\hline & & \multirow[b]{2}{*}{$D \_N P \_R$} & \multirow{2}{*}{\multicolumn{2}{|c|}{$D N R$}} & \multicolumn{3}{|c|}{$D$ : Variable muette année de crise } \\
\hline & & & & & & $D \_N P R$ & $D \_N R$ \\
\hline Constante & $\begin{array}{l}-0.040 \\
-2.465 \\
\end{array}$ & & & & & & \\
\hline$V E_{i, m-1}$ & $\begin{array}{l}-0.496 \\
-2.885\end{array}$ & & & & $\begin{array}{l}0.002 \\
0.008\end{array}$ & & \\
\hline$V E_{i, m-2}$ & $\begin{array}{l}-0.253 \\
-2.628 \\
\end{array}$ & & & & $\begin{array}{r}0.025 \\
0.179\end{array}$ & & \\
\hline$V E_{i, m-3}$ & $\begin{array}{l}-0.116 \\
-2.427\end{array}$ & & & & $\begin{array}{l}0.048 \\
0.615\end{array}$ & & \\
\hline$R_{i, m}$ & $\begin{array}{l}0.018 \\
3.236\end{array}$ & $\begin{array}{l}0.034 \\
1.409\end{array}$ & & $\begin{array}{l}013 \\
666\end{array}$ & $\begin{array}{l}-0.024 \\
-0.940\end{array}$ & $\begin{array}{l}-0.025 \\
-0.222\end{array}$ & $\begin{array}{l}0.012 \\
0.419\end{array}$ \\
\hline$R_{i, m-1}$ & $\begin{array}{l}0.032 \\
5.353\end{array}$ & $\begin{array}{l}-0.022 \\
-0.864\end{array}$ & & $\begin{array}{l}017 \\
506\end{array}$ & $\begin{array}{l}0.040 \\
3.476\end{array}$ & $\begin{array}{l}0.234 \\
2.965\end{array}$ & $\begin{array}{l}-0.082 \\
-3.232\end{array}$ \\
\hline$R_{i, m-2}$ & $\begin{array}{l}0.026 \\
5.438\end{array}$ & $\begin{array}{l}-0.027 \\
-2.240\end{array}$ & & $\begin{array}{l}012 \\
234\end{array}$ & $\begin{array}{l}0.034 \\
1.977\end{array}$ & $\begin{array}{l}0.077 \\
3.003\end{array}$ & $\begin{array}{l}-0.029 \\
-1.520\end{array}$ \\
\hline$R_{i, m-3}$ & $\begin{array}{l}0.018 \\
2.534 \\
\end{array}$ & $\begin{array}{c}0.014 \\
0.940 \\
\end{array}$ & & $\begin{array}{l}006 \\
189 \\
\end{array}$ & $\begin{array}{r}0.045 \\
3.124 \\
\end{array}$ & $\begin{array}{r}-0.013 \\
-0.168 \\
\end{array}$ & $\begin{array}{l}-0.029 \\
-1.900\end{array}$ \\
\hline \multicolumn{2}{|c|}{ Nombre d'observations } & \multicolumn{2}{|c|}{13086} & & & & \\
\hline \multicolumn{2}{|c|}{ Statistique de Fisher } & \multicolumn{2}{|c|}{5.265} & $\begin{array}{c}p \text { value } \\
(0.000)\end{array}$ & & & \\
\hline \multicolumn{2}{|l|}{ R2 ajusté } & \multicolumn{2}{|c|}{0.201} & & & & \\
\hline
\end{tabular}

